LBC group than in the conventional-cytology group, mainly because fewer LBC results were obscured by inflammation. The sensitivities of LBC for the detection of cervical intraepithelial neoplasia of grade 2 or more and of grade 3 or more were not significantly different from those of conventional cytology; however, the positive predictive value of LBC for grade 2 or more neoplasia was considerably lower than that of conventional cytology (relative positive predictive value $0.58,95 \% \mathrm{Cl} 0.44-0.77$ ). Finally, LBC detected more lesions of grade 1 or more than did conventional cytology (relative sensitivity $1.68,95 \% \mathrm{Cl} 1.40-2.02$ ), with a particularly marked increase in the detection of such lesions noted in women aged 25-34 years.

The authors conclude that the sensitivity of LBC is not significantly different to that of conventional cytology, although the positive predictive value of the former technique is lower. They suggest that the main benefit of switching to LBC screening would be a reduction in the number of unsatisfactory slides.

Original article Ronco G et al. (2007) Accuracy of liquid based versus conventional cytology: overall results of new technologies for cervical cancer screening: randomised controlled trial. BMJ 335: 28-31

\section{Development of rash indicates positive outcome in erlotinib- treated patients}

Patients treated with EGFR inhibitors frequently develop a rash. Previous studies have suggested a positive association between outcome and rash development. A study by Wacker et al. has examined the correlation between the clinical efficacy of EGFR inhibitors and the development of rash using data from two phase III studies.

The BR.21 study included 444 erlotinibtreated and 229 control patients with non-smallcell lung cancer. Patients with grade 1 rash survived $144 \%$ longer than patients who did not develop rash (hazard ratio $[\mathrm{HR}] 0.41 ; P<0.001$ ), while patients who developed a rash of grade $\geq 2$ survived $245 \%$ longer than did patients with no rash (HR 0.29; $P<0.001)$. Progression-free survival also correlated with the occurrence and severity of rash (HR 0.51, $P<0.001$ for grade 1 rash; HR 0.35, $P<0.001$ for grade 2 rash). In the erlotinib-treated group, disease control rate increased with the development and severity of rash $(P<0.001$ for both grade 1 and 2 rash versus grade 0). The PA.3 study included 254 patients with pancreatic cancer treated with erlotinib plus gemcitabine and 245 patients who received only gemcitabine (control group). In comparison with controls, only patients with grade $\geq 2$ rash had improved overall survival $(\mathrm{HR}=0.47$; $P<0.001)$, progression-free survival $(\mathrm{HR}=0.43$; $P<0.001)$, and disease control $(P=0.002)$.

The authors conclude that development of rash should be considered an indicator of a positive clinical outcome in erlotinib-treated patients.

Original article Wacker B et al. (2007) Correlation between development of rash and efficacy in patients treated with the epidermal growth factor receptor tyrosine kinase inhibitor erlotinib in two large phase III studies. Clin Cancer Res 13: 3913-3921

\section{Poor results with intensified maintenance chemotherapy in acute promyelocytic leukemia}

A prospective, multicenter study by the Japan Adult Leukemia Study Group, JALSG APL97, has shown that moderately intensive intermittent maintenance chemotherapy is detrimental to the survival of patients with acute promyelocytic leukemia (APL) who have become negative for the $P M L-R A R \alpha$ transcript after intensive consolidation chemotherapy.

Adults with previously untreated newly diagnosed APL were given induction therapy with all-trans retinoic acid alone or in conjunction with chemotherapy. A high proportion (94.3\%) of the 283 evaluable individuals achieved complete remission. Predicted 6-year overall and disease-free survival rates were $83.9 \%$ of all assessable cases and $68.5 \%$ of patients in complete remission, respectively. Patients with complete remission received three cycles of consolidation chemotherapy. Suitable patients who were negative for $P M L-R A R \alpha$ after completion of consolidation were randomized to receive six cycles of intensified maintenance chemotherapy every 6 weeks $(n=89)$ or observation $(n=86)$.

Median follow-up after randomization was 49 months. Predicted 6-year disease-free survival rates were similar with intensified chemotherapy and observation $(63.1 \%$ and $79.8 \%$, respectively; $P=0.20$ ), but predicted 6 -year overall survival was significantly lower with treatment $(86.2 \%$ vs $98.8 \% ; P=0.014)$. A large number of late ( $>3$ years) relapses in 